\title{
Light element synthesis constraining the supernova neutrino spectrum
}

\author{
Takashi Yoshida ${ }^{1}$, Toshitaka Kajino ${ }^{2,3}$, and Dieter H. Hartmann ${ }^{4}$ \\ ${ }^{1}$ Astronomical Institute, Graduate School of Science, Tohoku University, Aramaki, Aoba-ku, \\ Sendai 980-8578, Japan email: tyoshida@astr.tohoku.ac.jp \\ ${ }^{2}$ National Astronomical Observatory, and The Graduate University for Advanced Studies, \\ 2-21-1 Osawa, Mitaka, Tokyo 181-8588, Japan \\ ${ }^{3}$ Department of Astronomy, Graduate School of Science, University of Tokyo, 7-3-1 Hongo, \\ Bunkyo-ku, Tokyo 113-0033, Japan \\ ${ }^{4}$ Department of Physics and Astronomy, Clemson University, Clemson, SC29634, USA
}

\begin{abstract}
We constrain energy spectra of supernova neutrinos using the $\nu$-process light element synthesis in supernovae and the ${ }^{11} \mathrm{~B}$ abundance during Galactic chemical evolution. We calculate supernova nucleosynthesis due to the $\nu$-process assuming that neutrino energy spectra are FermiDirac distributions with zero chemical potential. We investigate the dependence of the ${ }^{11} \mathrm{~B}$ yield on the total neutrino energy and the temperature of $\nu_{\mu, \tau}$ and $\bar{\nu}_{\mu, \tau}$. From the obtained yields and the contribution to the ${ }^{11} \mathrm{~B}$ yield from supernovae constrained by observed abundances and Galactic chemical evolution models, we find an acceptable range of the temperature of $\nu_{\mu, \tau}$ and $\bar{\nu}_{\mu, \tau}$ of $4.8 \mathrm{MeV}$ to $6.6 \mathrm{MeV}$.
\end{abstract}

Keywords. Supernovae: general, nuclear reactions, nucleosynthesis, abundances, Galaxy: evolution

\section{Introduction}

Supernovae (SNe) are one of the important sites for light element (Li-Be-B) production during Galactic chemical evolution (GCE). SNe provide mainly ${ }^{11} \mathrm{~B}$ and ${ }^{7} \mathrm{Li}$ through the neutrino-nucleus interaction, referred to as the $\nu$-process (Woosley, et al. 1990). Recent studies of GCE of light elements indicated that the contribution of ${ }^{11} \mathrm{~B}$ from $\mathrm{SNe}$ derived from explosive nucleosynthesis models (Woosley \& Weaver 1995; WW95) is larger by a factor of $2.5 \sim 5.6$ than that evaluated from GCE models (e.g., Fields, et al. 2000). However, the ${ }^{11} \mathrm{~B}$ and ${ }^{7} \mathrm{Li}$ yields depend on supernova neutrino parameters which have not yet been precisely determined. We investigate the dependence of the ${ }^{11} \mathrm{~B}$ and ${ }^{7} \mathrm{Li}$ yields in SNe on the total neutrino energy and the neutrino energy spectra. Then, we constrain the SN neutrino parameters through limitations on the ${ }^{11} \mathrm{~B}$ yield determined by input from GCE.

\section{Supernova model}

We assume that the energy spectra of SN neutrinos obey the form of Fermi-Dirac distributions with zero chemical potentials. The temperatures of $\nu_{\mathrm{e}}$ and $\bar{\nu}_{\mathrm{e}}$ are set to be $3.2 \mathrm{MeV}$ and $5.0 \mathrm{MeV}$. The neutrino luminosity decreases exponentially with time with the decay time of $\sim 3 \mathrm{~s}$. We treat the total neutrino energy $E_{\nu}$ and the temperature of $\nu_{\mu, \tau}$ and $\bar{\nu}_{\mu, \tau}, T_{\nu_{\mu, \tau}}$, as free parameters. We use a $16.2 M_{\odot}$ presupernova star corresponding to SN 1987A (Shigeyama \& Nomoto 1990) as a progenitor model. The 

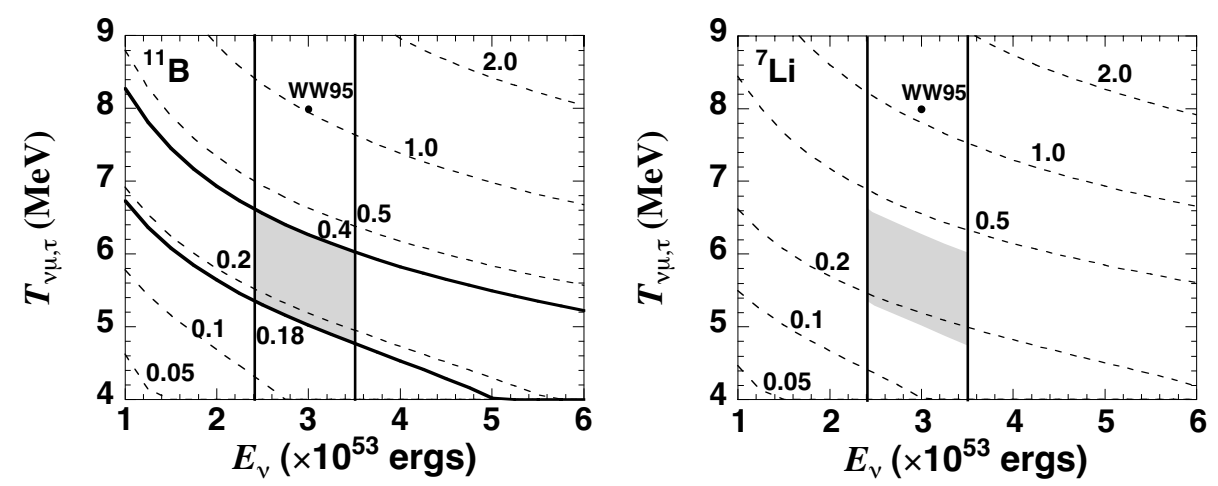

Figure 1. Contour lines of overproduction factor $f_{\nu}$ in the parameter plane of $E_{\nu}$ and $T_{\nu \mu, \tau}$. The region between two vertical lines indicates the gravitational energy range relevant for $\sim 1.4 M_{\odot}$ neutron star. The point labeled WW95 indicates the specific parameter values used in WW95. The region between the two solid contour lines in ${ }^{11} \mathrm{~B}$ panel is the $f_{\nu}$ range appropriate for GCE of ${ }^{11} \mathrm{~B}$. The shaded region is the part of parameter space in which both constraints $\left(\mathrm{GCE}{ }^{11} \mathrm{~B}\right.$ yield and neutron star binding energy) are simultaneously satisfied. A similar box is drawn for the case of ${ }^{7} \mathrm{Li}$.

supernova explosion is calculated with the explosion energy of $1 \times 10^{51}$ ergs and the mass cut of $1.6 M_{\odot}$. Detailed nucleosynthesis during the explosion is calculated using a nuclear reaction network containing 291 nuclear species (Yoshida, et al. 2004).

\section{Results}

In our model, ${ }^{11} \mathrm{~B}$ and ${ }^{7} \mathrm{Li}$ are mainly produced in the He layer. In there, ${ }^{7} \mathrm{Li}$ is produced through ${ }^{4} \mathrm{He}\left(\nu, \nu^{\prime} p\right)^{3} \mathrm{H}(\alpha, \gamma){ }^{7} \mathrm{Li}$ and ${ }^{4} \mathrm{He}\left(\nu, \nu^{\prime} n\right){ }^{3} \mathrm{H}(\alpha, \gamma){ }^{7} \mathrm{Be}\left(\mathrm{e}^{-}, \nu_{\mathrm{e}}\right)^{7} \mathrm{Li}$. Most of ${ }^{11} \mathrm{~B}$ is produced through ${ }^{7} \mathrm{Li}(\alpha, \gamma){ }^{11} \mathrm{~B}$. Less abundant ${ }^{11} \mathrm{~B}$ is produced through ${ }^{12} \mathrm{C}\left(\nu, \nu^{\prime} p\right){ }^{11} \mathrm{~B}$ and ${ }^{12} \mathrm{C}\left(\nu, \nu^{\prime} n\right){ }^{11} \mathrm{C}\left(\beta^{+}\right){ }^{11} \mathrm{~B}$ in the $\mathrm{O} / \mathrm{C}$ layer. When we set the total neutrino energy $E_{\nu}$ to $3 \times 10^{53}$ ergs and the neutrino temperature $T_{\nu_{\mu} \tau}$ equal to $6 \mathrm{MeV}$, the ${ }^{11} \mathrm{~B}$ and ${ }^{7} \mathrm{Li}$ yields are $1.92 \times 10^{-6} M_{\odot}$ and $7.37 \times 10^{-7}$. They are consistent with the yields of S20A model in WW95; the ${ }^{11} \mathrm{~B}$ and ${ }^{7} \mathrm{Li}$ yields are $1.85 \times 10^{-6} M_{\odot}$ and $6.67 \times 10^{-7} M_{\odot}$.

We constrain the neutrino temperature $T_{\nu_{\mu, \tau}}$ from GCE models of ${ }^{11} \mathrm{~B}$ abundance and the constraint on the total neutrino energy. Figure 1 shows the dependence of the ${ }^{11} \mathrm{~B}$ and ${ }^{7} \mathrm{Li}$ yields in our model on the total neutrino energy $E_{\nu}$ and the neutrino temperature $T_{\nu_{\mu, \tau}}$. The overproduction factor $f_{\nu}$ is defined by the ratios of the yields of ${ }^{11} \mathrm{~B}$ and ${ }^{7} \mathrm{Li}$ to the corresponding yields presented in WW95. We evaluate the range of $f_{\nu}$ from GCE models of ${ }^{11} \mathrm{~B}$ abundance (e.g., Fields, et al. 2000) as $0.18<f_{\nu}<0.40$. The appropriate range of $E_{\nu}$ is evaluated as $2.4 \times 10^{53}$ ergs $<E_{\nu}<3.5 \times 10^{53}$ ergs from the gravitational energy of $\mathrm{a} \sim 1.4 M_{\odot}$ neutron star (Lattimer \& Prakash 2001). Therefore, the neutrino temperature range reproducing the $\mathrm{SN}$ contribution of ${ }^{11} \mathrm{~B}$ in $\mathrm{GCE}$ is 4.8 $\mathrm{MeV}<T_{\nu_{\mu, \tau}}<6.6 \mathrm{MeV}$; lower neutrino temperature is favorable. From this neutrino temperature range, we also constrain the ${ }^{7} \mathrm{Li}$ yield in a $\sim 20 M_{\odot}$ star $\mathrm{SN}$ between $1.3 \times$ $10^{-7} M_{\odot}$ and $2.9 \times 10^{-7} M_{\odot}$. We have also investigated the effect of nonzero chemical potential of the neutrino energy spectra on the production of ${ }^{11} \mathrm{~B}$ (see Yoshida, et al. $(2005))$.

\section{Acknowledgements}

This work has been supported by COE Research "Exploring New Science by Bridging Particle-Matter Hierarchy" in Graduate School of Science, Tohoku University (22160028), 
by the Ministry of Education, Culture, Sports, Science and Technology, Grants-in-Aid for Specially Promoted Research (13002001), and by the Mitsubishi Foundation.

\section{References}

Fields, B.D., Olive, K.A., Vangioni-Flam, E. \& Cassé, M. 2000, Astrophys J. 540, 930

Lattimer, J.M. \& Prakash, M. 2001, Astrophys J. 550, 426

Shigeyama, T. \& Nomoto, K. 1990, Astrophys J. 360, 242

Woosley, S.E., Hartmann, D.H., Hoffman, R.D. \& Haxton, W.C. 1990, Astrophys J. 356, 272

Woosley, S.E. \& Weaver, T.A. 1995, Astrophys J. Suppl. 101, 181 (WW95)

Yoshida, T., Kajino, T. \& Hartmann, D.H. 2005, Phys. Rev. Lett. 94, 231101

Yoshida, T., Terasawa, M., Kajino, T. \& Sumiyoshi, K. 2004, Astrophys J. 600, 204 


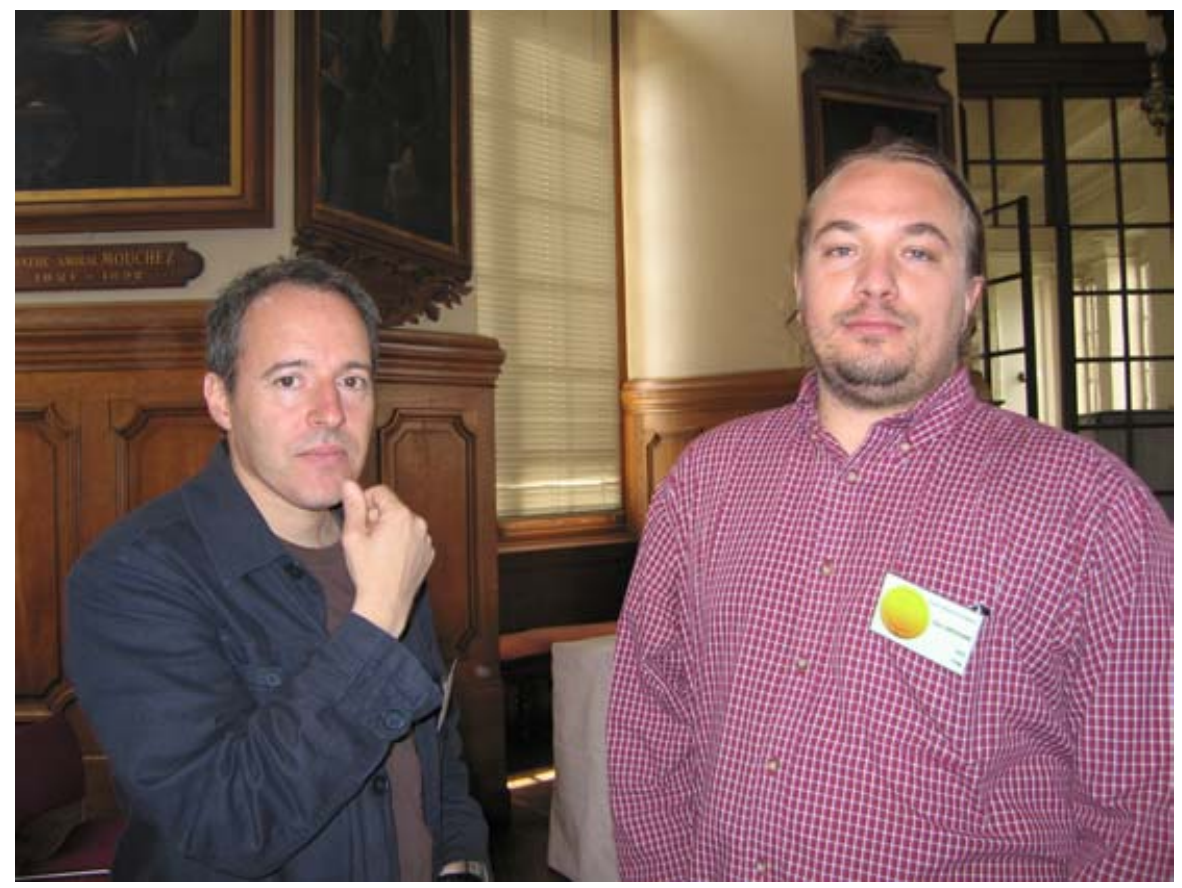

Patrick François and Eric Depagne (LOC) at the welcome reception.

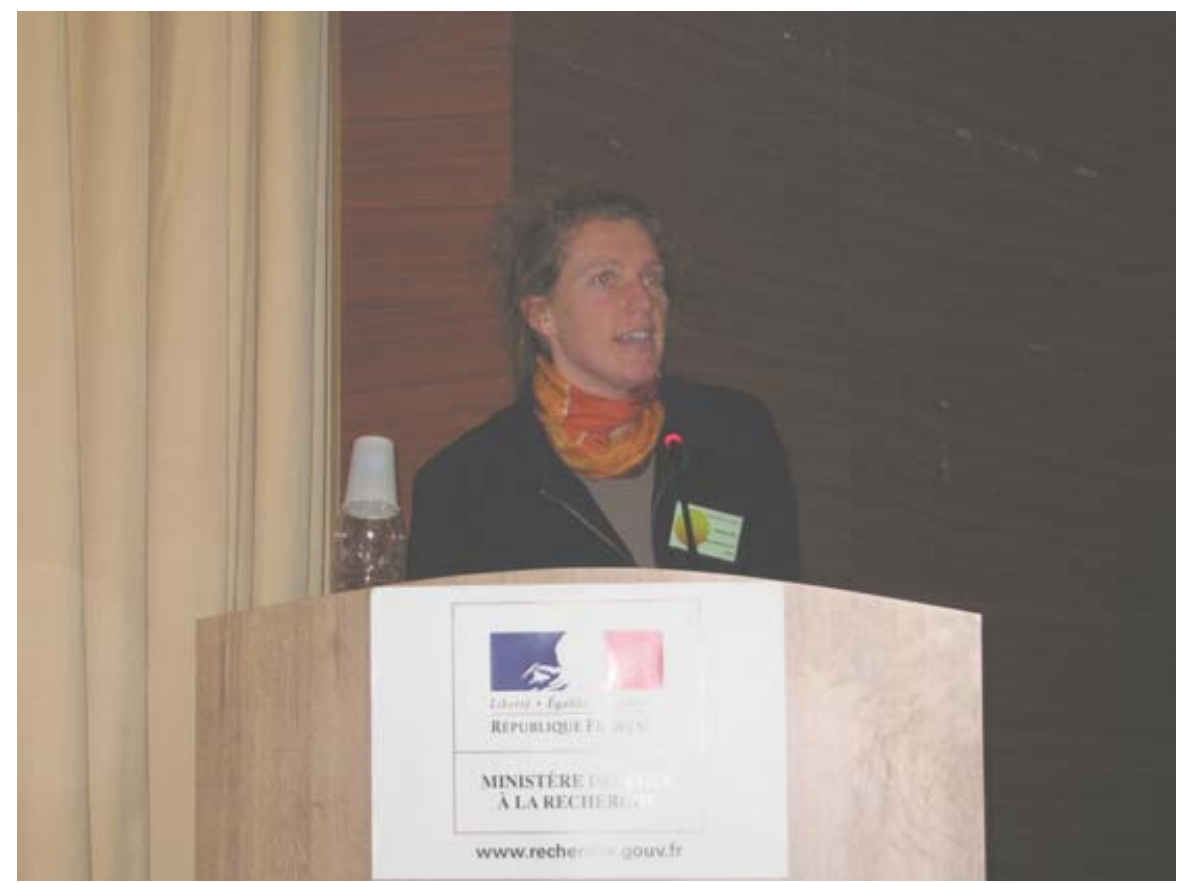

One of the many LOC annoucements at the conference, by Vanessa Hill. 